\title{
Controlling the Resistivity of Multi-walled Carbon Nanotube Networks by Copper Encapsulation
}

\author{
Yong Sun*, Boateng Onwona-Agyeman, and Tatsuro Miyasato ${ }^{1}$ \\ *Tel.: +81 93884 3564; fax: +81 93884 3560; sun@ele.kyutech.ac.jp \\ Department of Applied Science for Integrated System Engineering, Kyushu Institute of \\ Technology, 1-1 Sensui-machi, Tobata, Kitakyushu-City, Fukuoka 804-8550, Japan \\ ${ }^{1}$ Research and Development Center for Higher Education, Nagasaki University. 1-14 \\ Bunkyou-Machi, Nagasaki-City, Nagasaki 852-5821, Japan
}

\begin{abstract}
The resistivity of multi-walled carbon nanotube networks can be changed by filling $\mathrm{Cu}$ into the nanotubes as well as by preparing the nanotube networks with various densities. The resistivity can be controlled to be lower than the c-axis resistivity of graphite, and has a low temperature coefficient of $-1.12 \times 10^{-5} / \mathrm{K}$ over the temperature range of $20 \sim 500 \mathrm{~K}$. Filling $\mathrm{Cu}$ into the nanotubes decreases the intra-tube resistivity, but the temperature coefficient of the resistivity is governed by the inter-tube resistivity of the nanotube network. Keywords: multi-walled carbon nanotube network; copper encapsulation; electrical resistivity; temperature coefficient
\end{abstract}




\section{Introduction}

Carbon nanotubes (CNTs) are being used extensively for electronic applications such as in optoelectronic devices, transistors, touch-screens, flexible microelectronics, and chemical sensors [1]. In such applications, the CNTs are used in forms such as single tubes, bundles, random networks, and composites with polymers, metals, or ceramics. When CNTs are used in a device, foreign atoms will adsorb onto their internal and external surfaces, and this leads to degradation of their electronic properties of the device if the CNTs are single-walled carbon nanotubes (SWCNTs). For this reason, multi-walled carbon nanotubes (MWCNTs) are more suitable for the device applications than SWCNTs because the inner graphene walls of MWCNTs can remain unreacted and the essential electronic structures can be retained.

SWCNT films are attractive replacements for transparent conductors such as tin-doped indium oxide (ITO) and fluorine-doped tin oxide (FTO) in optoelectronic devices [2]. However, their room-temperature conductivity decreases by about four orders of magnitude as thickness decreases from 100 to $1 \mathrm{~nm}$ [3]. The resistance of the SWCNT films is dominated by the resistance of the intertube junctions formed from the crossed semiconducting SWCNTs. On the other hand, the electrical and optical properties of MWCNT films related to their thickness are still not studied in detail. Moreover, it is highly possible to achieve low resistivity MWCNT films with reduced monolayer density by filling high-conducting metals into the MWCNTs resulting in a high transparency of MWCNT network as well.

It is reported that the conductance of an individual MWCNT is lower than that of a SWCNT because the conductance is only formed at the outermost wall of the MWCNT and also, the interaction of the tube walls decreases the conductance. But recent results reveal a substantial contribution of conduction from the second wall after carriers tunnel from the outermost wall [4]. A perfect electrical contact with an MWCNT can be achieved by preparing the contact electrodes 
during the tube growth and this technique has shown that the conductivity of MWCNT can be several hundred times higher than that of SWCNT [5]. From the above results, new application fields based on MWCNT networks can be developed by controlling the contact properties of the MWCNTs with metals as well as by filling metals into the nanotubes.

In this study, we have prepared copper-filled MWCNT networks at various $\mathrm{Cu}$ concentrations, and the resistivity of the network prepared with various densities is measured as a function of temperature. Carrier transport mechanisms in the various networks are also discussed.

\section{Experimental procedure}

Laser vaporization of a carbon target containing $\mathrm{Cu}$ was carried out in an argon gas atmosphere at a pressure of $0.5 \mathrm{MPa}$. A continuous wave $\mathrm{Nd}$ :YAG laser was used to vaporize the $\mathrm{Cu} /$ carbon target at room temperature. The Cu-filled MWCNT (Cu@MWCNT) powders obtained were characterized by field emission scanning electron microscope (FE-SEM; JEOL JSM-7000), transmission electron microscope (TEM; HITACHI H-9000NAR), and X-ray diffraction (XRD; JEOL JDX-3500K). The compositions of $\mathrm{C}$ and $\mathrm{Cu}$ in the network are obtained by energy dispersive X-ray spectrometry (EDS) analysis during the SEM observation. The $\mathrm{Cu} @$ MWCNT powders were pressed into a pellet at room temperature and in vacuum at the pressures of 50,76, 128, and $191 \mathrm{MPa}$ for $30 \mathrm{~min}$. The resultant pellets were $15 \mathrm{~mm}$ in diameter and $5 \mathrm{~mm}$ thick. Resistivity of the pellets was measured by means of four-point probe technique using a resistance tester (HIOKI 3541) with a resolution of 0.1 $\mu \Omega$. The pellet was set in a chamber with a residual gas pressure of less than $10^{-5} \mathrm{~Pa}$. The measurements were carried out in the temperature range of $20 \sim 500 \mathrm{~K}$ in steps of $1 \mathrm{~K}$ during heating and cooling at a rate of $0.14 \mathrm{~K} \mathrm{~min}^{-1}$. 


\section{Results and discussion}

The SEM image of a typical Cu@MWCNT pellet is shown in Fig. 1(a). The nanotubes with uniform diameter and high aspect-ratio, which constitute a random network, can be seen. From the SEM image, it is suggested that the resistance of the network will be determined by the total resistance of the inter- and intra-tubes as the filling of copper changes only the resistance of the intra-tube.

Figure 1(b) is a TEM image of the network showing filled and hollow tubes with an average diameter of about $20 \mathrm{~nm}$ as well as individual and large particles in the network. The analyses using electron beam diffraction showed that the hollow tube has the structure of graphite. The filled tube is $\mathrm{Cu} @ \mathrm{MWCNT}$, and the large particles are rare $\mathrm{Cu}$ crystals, or are covered by graphite layer. The electron beam diffraction patterns of both a filled tube and a particle are shown in Figs. 1(c) and 1(d), respectively. For the filled tube in Fig. 1(c), the diffraction shows patterns from graphite (200) planes as well as the (111), (200), (220) and (311) planes of the face-centred cubic (fcc) $\mathrm{Cu}$ are observed. The result indicates that the filled tubes in the network are the MWCNTs filled by crystalline $\mathrm{Cu}$. On the other hand, the diffraction spots from (111), (200), and (220) planes of copper show that the large particles in the network have a single-crystal $\mathrm{Cu}$ structure.

The Cu@MWCNT network of the pellet is also analyzed by X-ray diffraction. Diffraction patterns of the Cu@MWCNT powders exposed to air for one and nine weeks are shown in Fig. 2(a) and 2(b), respectively. Similar to the results obtained by the electron beam diffraction, the XRD patterns in Fig. 2 shows the diffractions from graphite (200) plane as well as from the (111), (200), and (220) planes of the fcc $\mathrm{Cu}$ as well as a diffraction peak from $\mathrm{Cu}_{2} \mathrm{O}$ (111) planes. We found out that the intensity of the $\mathrm{Cu}_{2} \mathrm{O}$ (111) peak depends on exposure time of the powders to air. The intensity ratios of the $\mathrm{Cu}_{2} \mathrm{O}(111)$ peak to the $\mathrm{Cu}(111)$ peak are 0.10 and 0.18 for the exposure times of one and nine weeks. This suggests that the single-crystal $\mathrm{Cu}$ particles in the network are 
rare or are covered by a defective graphite layer.

The temperature dependences of the resistivities of the $\mathrm{Cu}$-filled and pristine MWCNT network pellets prepared by applying pressure of $128 \mathrm{MPa}$ are shown in Fig. 3 during the heating and cooling processes. The upper and bottom curves in the figure are related to the pellets with the $\mathrm{C} / \mathrm{Cu}$ ratios of 0.73 and 0.57 , respectively. The larger $\mathrm{C} / \mathrm{Cu}$ ratio corresponds to a smaller filling yield of $\mathrm{Cu}$ into the tubes, and there is no significant difference on the dependences during the heating and cooling processes for the same pellet. The resistivity of the network decreases with increasing the $\mathrm{Cu}$ yield filled into the tubes. The resistivity is almost inversely proportional to the temperature with an average temperature coefficient of $-1.12 \times 10^{-5} / \mathrm{K}$ over the range of $20 \sim 500 \mathrm{~K}$. On the other hand, the curves in the middle, corresponding to the scale of the right y-axis, show the temperature dependences of pristine MWCNT network during the heating and cooling processes. The pristine MWCNT network shows larger resistivity, about a hundred times that of the Cu@MWCNT network. But, the value of temperature coefficient is close to that of the $\mathrm{Cu} @$ MWCNT network, therefore, the temperature coefficient of the $\mathrm{Cu} @ M W C N T$ network is governed by the inter-tube resistivity. The noisy conductivity of the pristine MWCNT in figure 3 may be due to transport processes of carrier through the inter-tube network involving scatterings caused by the adsorption and desorption of gases on the surfaces of the tubes. With the introduction of copper, conductive paths are created between the tubes reducing the scattering involving the gas and hence eliminating the noisy nature of the filled-MWCNT curves.

The room-temperature resistivity of the $\mathrm{Cu} @ \mathrm{MWCNT}$ pellets with the $\mathrm{C} / \mathrm{Cu}$ ratio of 0.57 is also measured for samples prepared at various pressures of 50, 76, 128, and $191 \mathrm{MPa}$. The room-temperature resistivity is shown in Fig. 4 as a function of pressure and it can be observed that resistivity decreases with increase in the applied pressure or the density of the network. Increasing the density of the $\mathrm{Cu} @$ MWCNT network is another means of effectively changing its resistivity apart from filling $\mathrm{Cu}$ into the tubes. 
Based on the above results, we can discuss the mechanisms of carrier transport in the $\mathrm{Cu} @$ MWCNT network. Firstly, the resistivities of the $\mathrm{Cu} @$ MWCNT network at $300 \mathrm{~K}$ are 0.042 and $0.028 \Omega \mathrm{cm}$ for the $\mathrm{C} / \mathrm{Cu}$ ration of 0.73 and 0.57 . The values are lower than the resistivity (1 $\Omega \mathrm{cm}[6])$ of MWCNT film at $300 \mathrm{~K}$, and are also lower than the c-axis resistivity $(0.1 \Omega \mathrm{cm}[7])$ of highly oriented pyrolytic graphite (HOPG) at $300 \mathrm{~K}$. This indicates that the filling of $\mathrm{Cu}$ decreases effectively the resistivity of the MWCNT network.

Secondly, the measured temperature coefficient of the $\mathrm{Cu} @$ MWCNT networks has smaller value of $-1.12 \times 10^{-5} / \mathrm{K}$ over the temperature range of $20 \sim 500 \mathrm{~K}$. This is due to the fact that the MWCNT has a negative temperature coefficient [6], and also as shown in Fig. 3. The result shows that, the filling of $\mathrm{Cu}$ can decrease the intra-tube resistivity of the $\mathrm{Cu} @$ MWCNT network.

Thirdly, the contact resistance between the metal and MWCNT depends upon tunneling of electrons across a finite physical barrier created by Van der Waals interaction at the metal/CNT interface [8]. In this study, the barrier height depends mainly on the work function difference between $\mathrm{Cu}$ and MWCNT. The work functions are $4.8 \mathrm{eV}$ for MWCNT [9], $4.48 \mathrm{eV}$ for $\mathrm{Cu}(110)$, $4.53 \mathrm{eV}$ for $\mathrm{Cu}(112), 4.59 \mathrm{eV}$ for $\mathrm{Cu}(100)$, and $4.95 \mathrm{eV}$ for $\mathrm{Cu}(111)$ surfaces [10]. The barrier leads to a contact resistance across the $\mathrm{Cu} / \mathrm{MWCNT}$ interface and in particular, if a smaller voltage is applied to the interface [9]. As seen in Fig. 3, therefore, the resistivity of the Cu@MWCNT network, which is lower than the c-axis resistivity of graphite, is related to the filling of $\mathrm{Cu}$ into the nano-tubes.

Finally, the resistivity of the $\mathrm{Cu@MWCNT} \mathrm{network} \mathrm{is} \mathrm{also} \mathrm{dependent} \mathrm{on} \mathrm{the} \mathrm{number} \mathrm{of}$ conductive paths through the network and the number of inter-tube junctions encountered on a given path. Higher density of the network results in the lowering of the resistivity as shown in Fig. 4. Filling $\mathrm{Cu}$ into the hollow tubes can only decrease the intra-tube resistivity by increasing the conducting cross-section of the tubes. 


\section{Conclusion}

In summary, the $\mathrm{Cu} @ \mathrm{MWCNT}$ network was prepared and its resistivity was measured as a function of temperature and density of the network. The resistivity can be lower than the c-axis resistivity of graphite and has a smaller and constant temperature coefficient over the temperature range from 20 to $500 \mathrm{~K}$. The resistivity decreases with increasing the $\mathrm{Cu}$ amount encapsulated into the tubes as well as the density of the network.

\section{Acknowledgments}

This work was partially supported by project No. 15-B01, Program of Research for the Promotion of Technological Seeds, Japan Science and Technology Agency. The work was also partially supported by Grant-in-Aid for Exploratory Research No:23651115, Japan Society for the Promotion of Science (JSPS). 


\section{References}

[1] Bandaru Prabhakar R. J. Nanosci. \& Nanotech. 2007; 7: 1239-1267.

[2] Wu ZC, Chen ZH, Du X, Logan JM, Sippel J, Nikolou M, Kamaras K, Reynolds JR, Tanner DB, Hebard AF, Rinzler AG. Science 2004; 305: 1273-1276.

[3] Bekyarova E, Itkis ME, Cabrera N, Zhao B, Yu A, Gao J, Haddon RC. J. Am. Chem. Soc. 2005; 127: 5990-5995.

[4] Bourlon B, Miko C, Forró L, Glattli DC, Bachtold A. Phys. Rev. Lett. 2004; 93: 176806(1-4).

[5] Li HJ, Lu WG, Li JJ, Bai XD, Gu CZ. Phys. Rev. Lett. 2005; 95: 086601(1-4).

[6] Heer WA, Bacsa WS, Chatelain A, Gerfin T, Humphrey-Baker R, Forro L, Ugarte D. Science 1995; 268: 845-847.

[7] Uher C, Hockey RL, Ben-Jacob E. Phys. Rev. B 1987; 35: 4483-4488.

[8] Rochefort A, Avouris P, Lesage F, Salahub DR. Phys. Rev. B 1999; 60: 13824-13830.

[9] Ngo Q, Petranovic D, Krishana S, Cassell AM, Ye Q, Li J, Meyyappan M, Yang CY. IEEE Trans. Nanotech. 2004; 3: 311-317.

[10] Gartland PO, Berge S, Slagsvold BJ. Phys. Rev. Lett. 1972; 28: 738-739. 


\section{Figure captions}

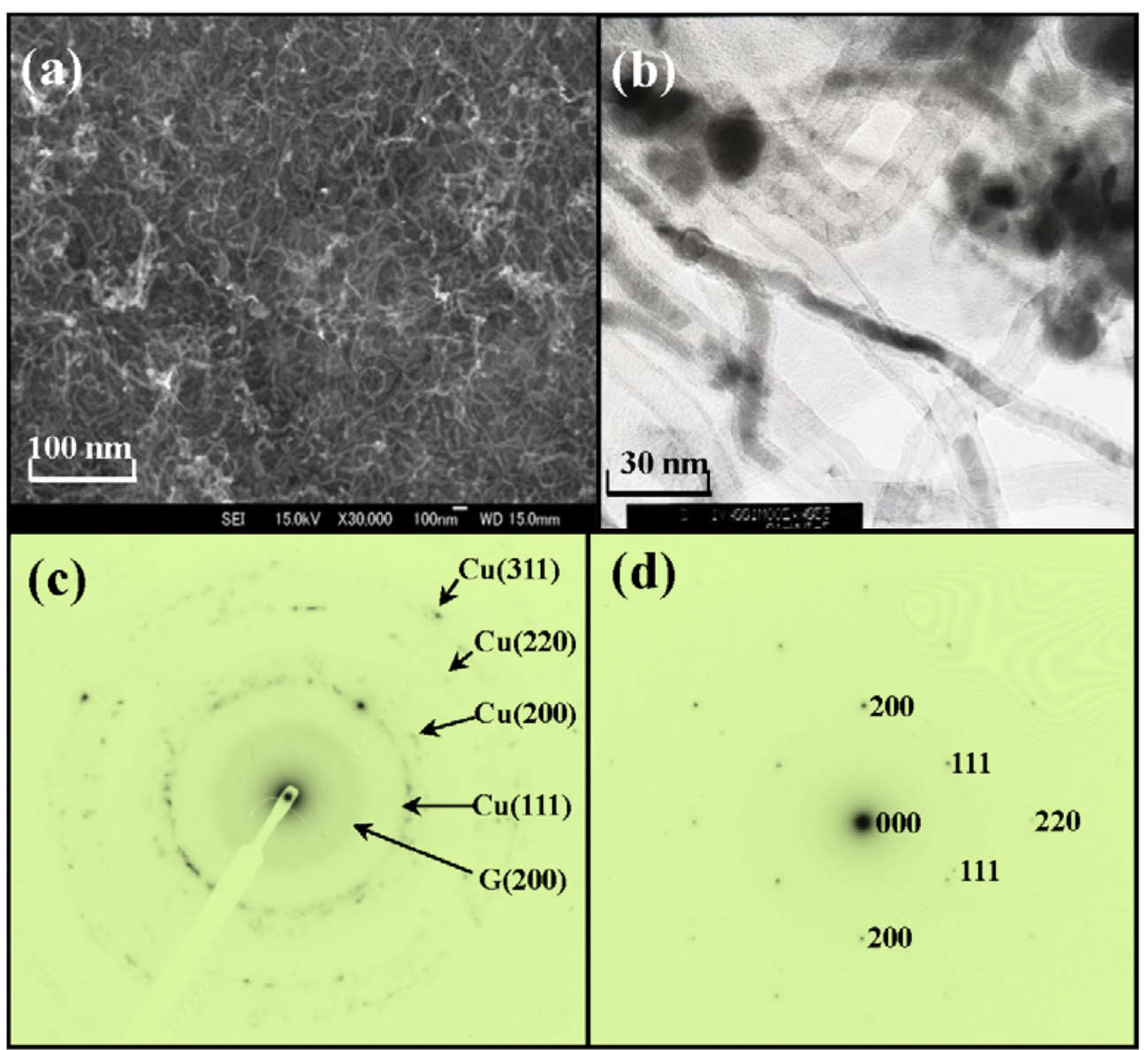

Fig. 1 (a) Typical SEM image of the Cu@MWCNT pellet, (b) TEM image of the Cu@MWCNT network, and electron beam diffraction patterns of: (c) filled tube and (d) a particle. 


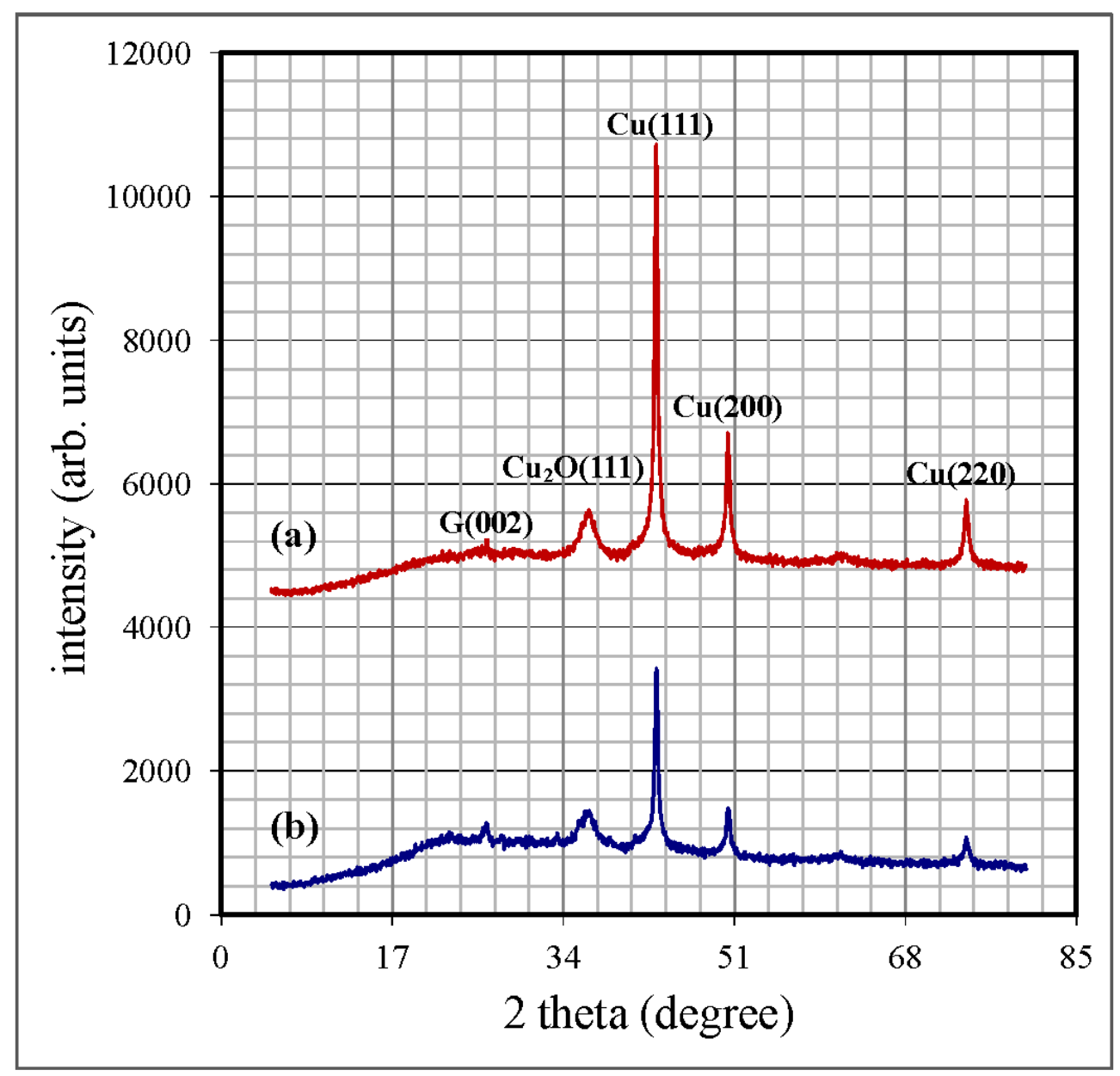

Fig. 2 XRD patterns of the Cu@MWCNT powders exposed to air for (a) one week and (b) nine weeks. 


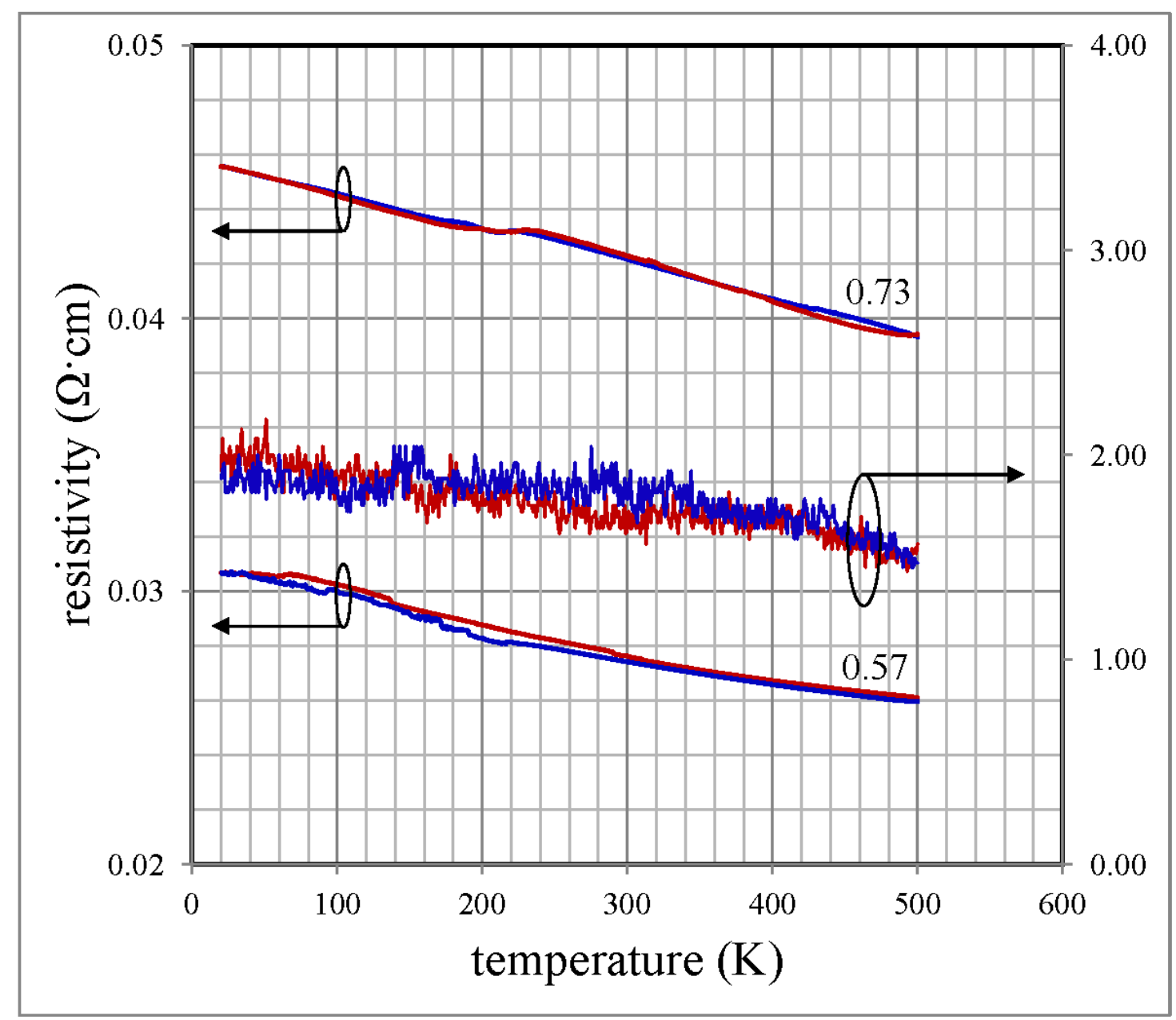

Fig. 3 Temperature dependences of the resistivities of the $\mathrm{Cu}$-filled and pristine MWCNT pellets prepared by applying pressure of $128 \mathrm{MPa}$ during heating and cooling. The upper and bottom curves are related to the pellets with the $\mathrm{C} / \mathrm{Cu}$ ratios of 0.73 and 0.57 , respectively. The curves in the middle shows temperature dependences of the resistivities of the pristine MWCNT pellet, corresponding to the right y-axis. 


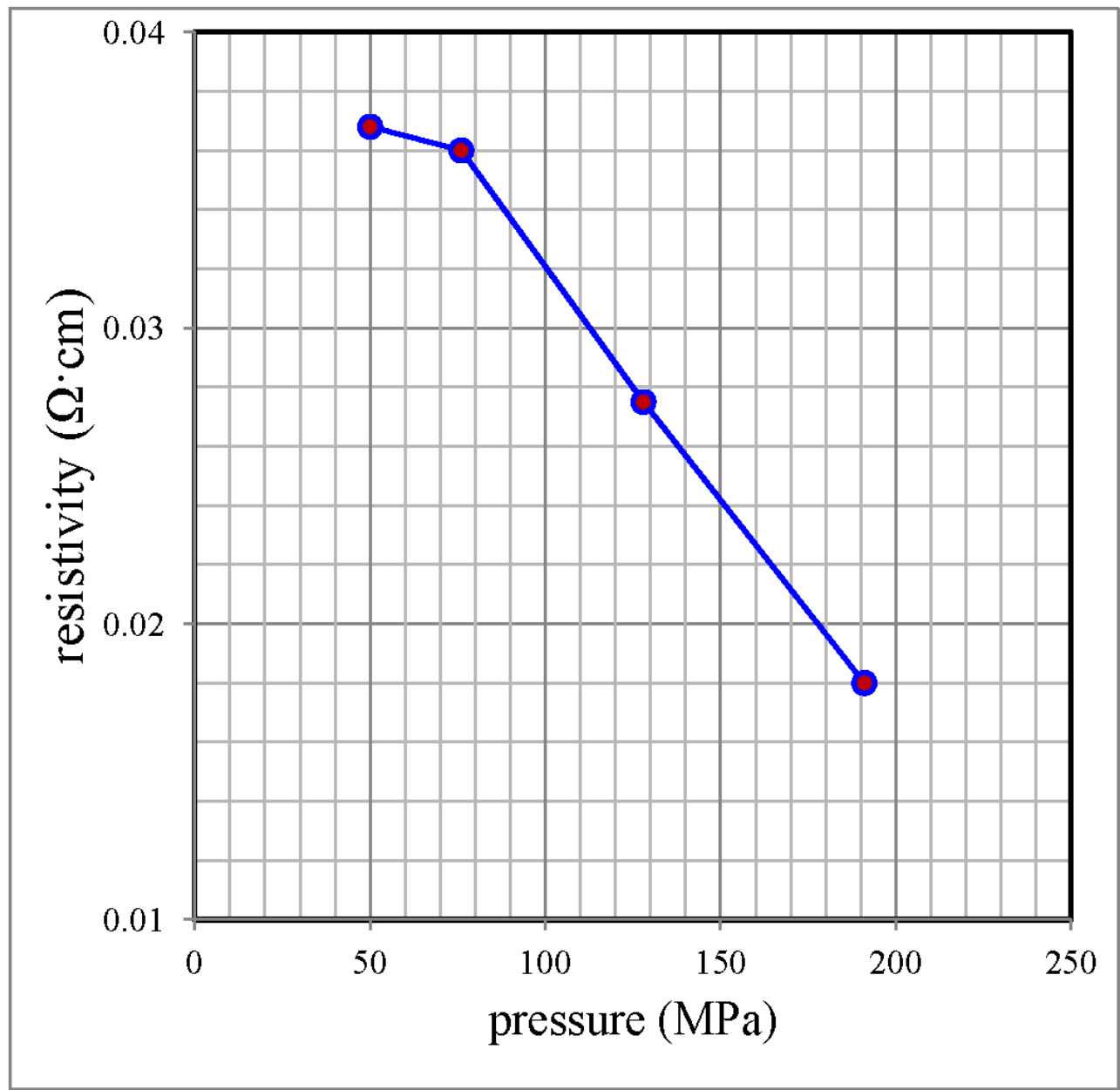

Fig. 4 Room-temperature resistivity of the $\mathrm{Cu} @$ MWCNT pellets with the $\mathrm{C} / \mathrm{Cu}$ ratio of 0.57 as a function of the pressure applied during pellet preparation. 